\title{
Mineralogical controls on mine drainage of the abandoned Ervedosa tin mine in north-eastern Portugal
}

\author{
M. Elisa P. Gomes *, Paulo J.C. Favas \\ Department of Geology, University of Trás-os-Montes e Alto Douro (UTAD), Ap.1013, 5000-801 Vila Real, Portugal
}

Available online 25 July 2006

\begin{abstract}
The Ervedosa Mine, in north-eastern Portugal, has Sn-bearing quartz veins containing cassiterite and sulphides that cut Silurian schists and a Sn-bearing muscovite granite. These veins were mined for $\mathrm{Sn}$ and $\mathrm{As}_{2} \mathrm{O}_{3}$ until 1969. Cassiterite, the main $\mathrm{Sn}$ ore, has alternate lighter and darker growth-zones. The darker zones are richer in $\mathrm{Fe}, \mathrm{Nb}, \mathrm{Ta}$ and $\mathrm{Ti}$, but poorer in Sn than the adjoining lighter zones. Exsolution blebs of ferrocolumbite, manganocolumbite, Ti ixiolite, rutile, ilmenite and rare wolframite were found in the darker zones. Arsenopyrite is the most abundant sulphide and contains inclusions of pyrrhotite, bismuth, bismuthinite and matildite. Other sulphides are pyrite, sphalerite, chalcopyrite and stannite. Secondary solid phases consisting mainly of hydrate sulphate complexes of $\mathrm{Al}, \mathrm{Fe}, \mathrm{Ca}$ and $\mathrm{Mg}$ (aluminocopiapite, copiapite, halotrichite, pickeringite, gypsum and alunogen, meta-alunogen) occur at the surface of the Sn-bearing quartz veins and their wall rocks (granite and schist), while oxides, hydroxides, arsenates and residual mineral phases (albite, muscovite and quartz) occur in mining tailings. Toxic acid mine waters (acid mine drainage AMD), which have high conductivity and significant concentrations of $\mathrm{As}, \mathrm{SO}_{4}$ and metal $(\mathrm{Cu}, \mathrm{Zn}, \mathrm{Pb}, \mathrm{Fe}, \mathrm{Mn}, \mathrm{Cd}, \mathrm{Ni}$ and $\mathrm{Co})$, occur in an area directly affected by the mine. Surface stream waters outside this area have low conductivity and a $\mathrm{pH}$ that is almost neutral. Metal and As concentrations are also lower. Stream waters within the impact area have an intermediate composition, falling between that of the AMD and the natural stream waters outside impact area. Waters associated directly with mineralised veins must not be used for human consumption or agriculture.
\end{abstract}

(C) 2006 Elsevier Ltd. All rights reserved.

\section{Introduction}

A major environmental issue associated with mining and mine waste is the release of As and heavy metals into the environment, often due to the unsatisfactory disposal of rock waste. When water infiltrates the rock waste, oxidation of sulphide minerals (especially pyrite) and their subsequent dissolution can result in acid mine water. Studies concerning acid mine drainage (AMD)

\footnotetext{
* Corresponding author.

E-mail address: mgomes@utad.pt (M.E.P. Gomes).
}

are increasing throughout the world, including those in many abandoned mines in Portugal (Antunes et al., 2002; Oliveira et al., 2002; Ávila, 2002; Valente, 2004). Elevated levels of As, greater than that allowed by current drinking-water regulations, in ground and surface water can be the result of purely natural phenomena; however, they are often due to anthropogenic activities such as mining and agriculture (Bednar et al., 2004). Effluents from abandoned mine workings typically consist of acid mine drainage, eroded material from mine tailings and waste from ore processing operations. 
Secondary minerals in efflorescent crusts on the surface of the waste pile or in the vein walls impinge on and attenuate the migration of As and metals from high-sulphide wastes (Gieré et al., 2003; Petrunic and Al, 2005). Secondary minerals play an important role in the natural retention of oxyanions $\left(\mathrm{H}_{2} \mathrm{AsO}_{4}^{-}\right)$and divalent cations $(\mathrm{Cu}, \mathrm{Zn}$ and $\mathrm{Pb})$, because some of these elements are captured by sorption and precipitation (Lee et al., 2005). Newly formed secondary minerals from AMD have been considered to play an important role in attenuating trace metals (Bigham et al., 1996; Webster et al., 1998; Valente, 2004).

The Ervedosa mine (also known as the Tuela mine), exploited in the past by the Phoenicians and the Romans, was in intermittent operation from 1928 to 1969; however, there has been no mining in the area since then. The last exploitation was underground, which continued upward into two large open pits; alluvial placers were also exploited. The tailings and rock waste contain concentrations of metals and were deposited on the ground and are at present partially covered by natural vegetation. Exposure of these materials to air and water produces acidic water from galleries and tailings, which facilitate the release of contaminants to aquatic ecosystems.

The aim of this study was to analyse the mineralogical control on acid mine drainage with respect to primary and secondary minerals and metal mobility. Water from mining galleries is compared with natural stream waters from inside and outside the mined area, using data collected over a period of one year.

\section{Geological setting and mineral paragenesis of the Ervedosa mine}

The Ervedosa Mine in north-eastern Portugal is in the schistose domain of the Galiza-Trás-os-Montes Zone of the Iberian Terrane. Here at Ervedosa, muscovite-biotite and muscovite granites intruded the Silurian phyllites and quartzites and Carboniferous biotite granitoids (Fig. 1b). A medium-grained porphyritic muscovite-biotite granite, a medium to fine-grained muscovite-biotite granite, and a fine to medium-grained muscovite granite define a sequence of in situ fractional crystallization (Gomes and Neiva, 2002). These granites yield a whole-rock $\mathrm{Rb}-\mathrm{Sr}$ isochron of $327 \pm 9 \mathrm{Ma}$ (Gomes and Neiva, 2002). The Sn-bearing quartz veins intrude the Silurian metasediments (425 Ma, Beetsma, 1995) and the muscovite granite (Fig. 1b). The mineralization is associated with the muscovite granite and was probably derived from the late stages of fractional crystallization of the granite suite because magmatic fractionation was responsible for the increase in $\mathrm{Sn}$ contents of granites and their micas (Gomes and Neiva, 2002).

Tin-bearing quartz veins are hosted in fractures that are related to the movements along a dextral $\mathrm{N} 30^{\circ} \mathrm{W}$-trending ductile shear zone (Fig. 1b). Cassiterite and sulphides are more common in $\mathrm{N} 70^{\circ} \mathrm{E}-$ trending, dominantly sub-vertical quartz veins of $<0.2 \mathrm{~m}$ in thickness, and sometimes in $\mathrm{N} 55^{\circ} \mathrm{E}$ $70^{\circ} \mathrm{S}$-striking quartz veins which are $0.2-0.8 \mathrm{~m}$ thick, whose lengths reach a $100 \mathrm{~m}$. Subhedral to euhedral cassiterites in quartz veins are generally less than $10 \mathrm{~mm}$ across and are locally cut by fractures filled with radial muscovite, quartz and arsenopyrite, chalcopyrite and stannite. Crystals of cassiterite are also locally found in round masses with a diameter of less than $10 \mathrm{~cm}$.

Three faulting hypogenic stages have been identified in these Sn-bearing quartz veins (Gomes and Neiva, 2001). A later stage is characterised by supergene phases - Fe oxides, U oxides, covellite and arsenates, observed in fine veins related to new fracture post-tectonic Hercynian faults. Barren quartz veins later cut into Sn-bearing veins. Very thin barren quartz veins, the so-called veinlets, cut the mineralised quartz veins and barren quartz veins.

\section{Methodology and analytical techniques}

Detailed studies of samples collected from outcrops in the mine and from boreholes included transmitted and reflected-light microscopy. These studies were carried out in order to establish the primary mineralogy and the paragenetic sequence (Gomes and Neiva, 2001).

The ore minerals were analysed on a Cámeca Camebax electron microprobe at INETI (ex. Instituto Geológico e Mineiro) S. Mamede de Infesta, Portugal. Analyses were conducted at an accelerating voltage of $15 \mathrm{kV}$ and a beam current of $20 \mathrm{nA}$. Standards used include cassiterite ( $\mathrm{Sn} \mathrm{L} \alpha), \mathrm{MnTiO}_{3}(\mathrm{Mn}$ $\mathrm{K} \alpha$ and $\mathrm{Ti} \mathrm{K} \alpha), \mathrm{Fe}_{2} \mathrm{O}_{3}(\mathrm{Fe} \mathrm{K} \alpha)$, sphalerite ( $\mathrm{Zn} \mathrm{K} \alpha$ and $\mathrm{S} \mathrm{k} \alpha)$, pyrite $(\mathrm{Fe} \mathrm{K} \alpha)$, galena $(\mathrm{Pb} \mathrm{M} \alpha)$, AsGa (As $\mathrm{K} \alpha), \mathrm{Cr}_{2} \mathrm{O}_{3}(\mathrm{Cr} \mathrm{K} \alpha)$, Ta (L $\left.\alpha\right), \mathrm{Nb}(\mathrm{L} \alpha)$, W (L $\left.\alpha\right)$, $\mathrm{Cd}(\mathrm{L} \alpha), \mathrm{Bi}(\mathrm{M} \alpha), \mathrm{Sb}(\mathrm{L} \alpha), \mathrm{Cu}(\mathrm{K} \alpha)$ and $\mathrm{Ag}(\mathrm{L} \alpha)$.

Identification of secondary minerals was performed by X-ray diffraction (XRD) using a Philips Diffractometer PW 1840 operating at $40 \mathrm{kV}$ and $25 \mathrm{~mA}$ at the University of Trás-os-Montes and 

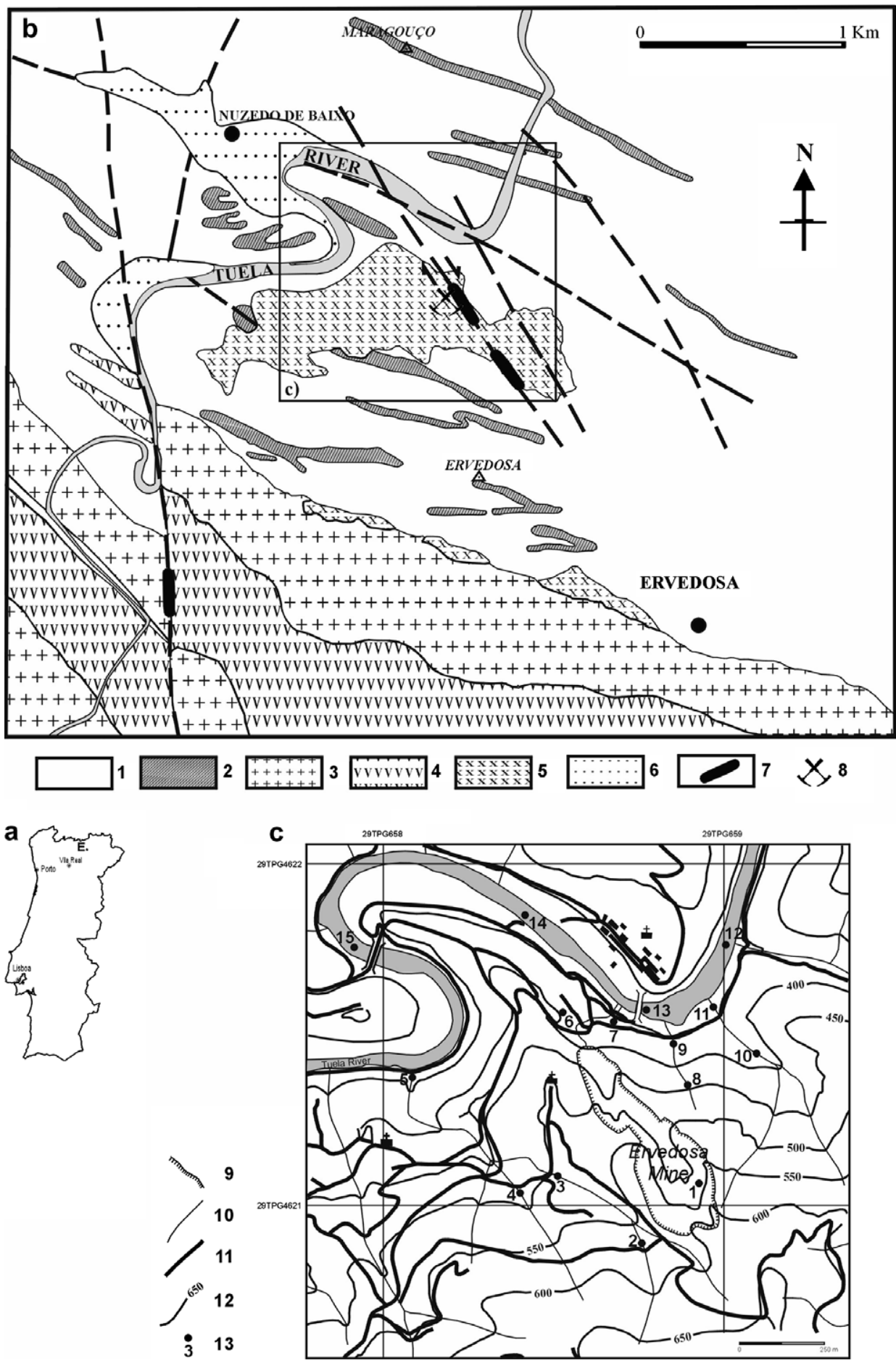

Fig. 1. (a) Location of the Ervedosa area on the map of Portugal; (b) Simplified geological map of abandoned Ervedosa mine; (c) Simplified topographical map with water sampling points. 1 - silurian pelitic metasedimentary rocks, 2 - silurian quartzites, 3 - medium to coarse-grained porphyritic biotite-muscovite granitoids, 4 - medium-grained slightly porphyritic muscovite-biotite granite, 5 - fine- to medium-grained muscovite granite, 6 - alluvium, 7 - quartz veins, 8 - abandoned Ervedosa Sn mines, 9 - exploitation limit, 10 - drainage lines, 11 - access routes, 12 - topography , 13 - samples number. 
Alto Douro (UTAD) in Portugal. Randomly oriented specimens were continuously scanned in the range $2-80^{\circ}, 2 \theta$ at a rate of $2^{\circ} / \mathrm{min}$, using $\mathrm{Cu} \mathrm{K \alpha}$ radiation $(\lambda=1.5419 \AA)$. Scanning Electron Microscope (SEM) instruments at UTAD at Vila Real and the University of Minho at Braga (Portugal) were used to investigate secondary minerals resulting from the oxidation of sulphide minerals, and to obtain backscattered electron images.

The exploitation area and the location of water samples are shown in Fig. 1c. AMD-contaminated surface water sampling points were located directly in abandoned mine workings, intermediate waters affected by mine workings or tailings drainage and natural stream water samples were collected outside these areas (Fig. 1c). Water was collected 6 times a year, every two months starting in February 2002. Some leachates are ephemeral, active only after rainfall episodes (November). Portable instruments were used to measure $\mathrm{pH}$, conductivity and temperatures in the field. The water was filtered, acidified and kept at $4{ }^{\circ} \mathrm{C}$. The laboratory analyses were performed at
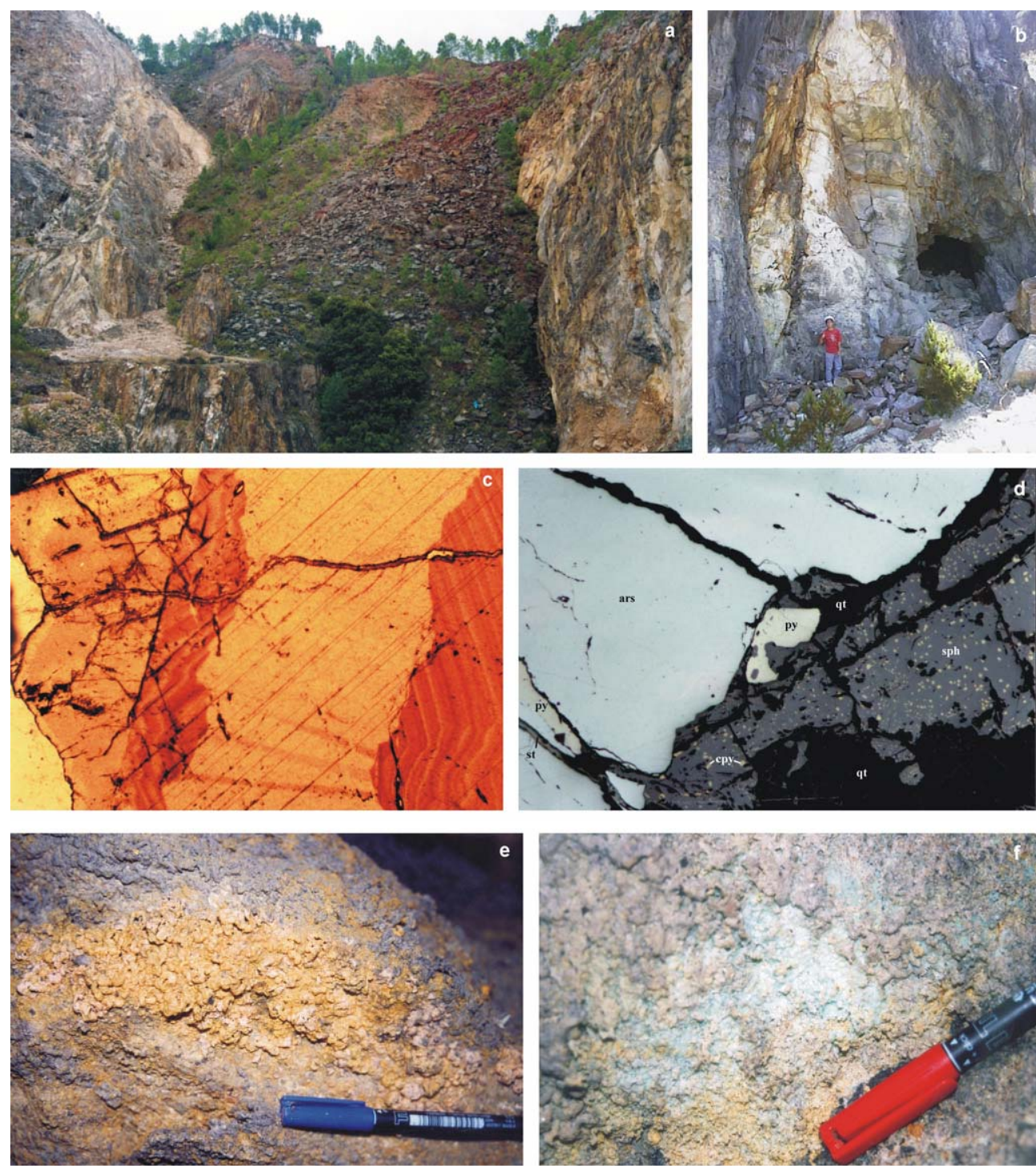

Fig. 2. (a) Ervedosa exploitation upward into two large open pits; (b) Highly deformed Sn-bearing quartz veins and entrance for gallery; (c) Irregularly zoned cassiterite crystals and exsolution products in the darker zones (polarized light, NP, $\times 40$ ); (d) Common sulphidesarsenopyrite (ars) cut by veinlets of stannite (st), pyrite (py), quartz (qt) and sphalerite (sph) with chalcopyrite belbs (cpy) (reflected light, $\times 40$ ); (e) Brown botryoidal efflorescences in schists; and (f) Greenish blue, white and brown efflorescences in granite. (For interpretation of the references to colour in this figure legend, the reader is referred to the web version of this article.) 
the Department of Earth Sciences, University of Coimbra (Portugal). Sulphate was analysed by gravimetry (APHA, 1995; Vetter et al., 1995). Sodium, $\mathrm{K}, \mathrm{Ca}$ and $\mathrm{Mg}$ were determined by spectrometry (Perkin-Elmer 303 flame atomic absorption), while for $\mathrm{Fe}, \mathrm{Cd}, \mathrm{As}, \mathrm{Ni}, \mathrm{Mn}, \mathrm{Cu}, \mathrm{Pb}, \mathrm{Zn}$ and $\mathrm{Co}$ a coupled graphite furnace was used.

\section{Results and discussion}

\subsection{Geochemistry of primary minerals}

As mentioned above, the last exploitation of the Ervadosa mine was underground, and continued upward into two large open pits (Fig. 2a). The lenticular shaped Sn-bearing quartz veins (Fig. 2b), which are sometimes highly deformed, contain cassiterite and rare stannite associated with sulphides whose mineralogical and geochemical characteristics were described by Gomes and Neiva (2001).

Crystals of cassiterite exhibit narrow and parallel, alternating lighter and darker growth-zones. In general the darker zones are strongly pleochroic $(\varepsilon-$ red, $\omega$-colorless) and have higher $\mathrm{Ta}, \mathrm{Nb}, \mathrm{Fe}$ and $\mathrm{Ti}$ and lower Sn contents than the lighter zones, which are nearly pure $\mathrm{SnO}_{2}$ (Fig. 2c, Table 1). Exsolution products, commonly columbite ranging from ferrocolumbite to manganocolumbite and ixiolite ranging from titanian ixiolite, $\mathrm{W} \geqslant \mathrm{Ti}$ - ixiolite and rarely niobian rutile, ilmenite and wolframite were mainly found in the darker zones of cassiterite (Fig. 2c, Table 1). Stannite rarely occurs with quartz and chalcopyrite in veinlets cutting cassiterite and arsenopyrite. The last sulphide mineral formed is stannite with the composition $\mathrm{Cu}_{1.9}\left(\mathrm{Fe}_{1.0} \mathrm{Zn}_{0.1}\right) \mathrm{SnS}_{4}$.

Other minerals are silicates (quartz and muscovite), sulphides, sulphosalts and oxides. The representative chemical compositions of sulphides and sulphosalts are given in Table 2. Arsenopyrite (FeAsS) is the most abundant sulphide mineral (Fig. 2d). It has inclusions of pyrrhotite $\left(\mathrm{Fe}_{0.9} \mathrm{~S}_{1.0}\right)$, native $\mathrm{Bi}$, bismuthinite $\left(\mathrm{Bi}_{2} \mathrm{~S}_{3}\right)$ and matildite $\left(\mathrm{AgBiS}_{2}\right)$. Arsenopyrite is replaced by pyrite $\left(\mathrm{FeS}_{2}\right)$, chalcopyrite $\left(\mathrm{CuFeS}_{2}\right)$, sphalerite $(\mathrm{ZnS})$ and stannite (Fig. 2d). In general, the latest anhedral arsenopyrite has relatively higher Fe content than the earliest euhedral to subhedral arsenopyrite. Later sphalerite is poorer in $\mathrm{Fe}$ than the earlier sphalerite. Matildite contains some additional $\mathrm{Fe}$ and $\mathrm{Pb}$ replacing $\mathrm{Ag}$.

\subsection{Mineralogy of secondary sulphate salts}

The soluble sulphate salts are usually found as botryoidal efflorescences in variable colours (white, greenish blue, pale green and yellow) suggesting a paragenetic sequence of sulphates with distinct solubilities and degrees of dehydration (Sánchez España et al., 2005) (Figs. 2e and f). Secondary phases are rarely found as monomineralic phases in the schist and granite walls of Ervedosa (Figs. 2e and $\mathrm{f}$ ), which are in close contact with Sn-bearing quartz veins as well as in some mineralized veins; these generally consist of mixtures of $\mathrm{Ca}$, $\mathrm{Al}, \mathrm{Fe}$ and $\mathrm{Mg}$ hydrated sulphates such as pickeringite, copiapite, halotrichite, alunogen, gypsum (Fig. 3). On the other hand, oxides, hydroxides, arsenates and residual mineral phases (albite, muscovite and quartz) occur in the mine tailings.

In the efflorescent crusts at the surface of the waste pile, As co-precipitates with the Fe(III) sulphate, copiapite; $\mathrm{Zn}$ and $\mathrm{Cu}$ are primarily incorporated into $\mathrm{Fe}(\mathrm{II})$ sulphates, and $\mathrm{Pb}$ is mainly co-precipitated with minerals of the jarosite group. Co-precipitation of toxic elements with sulphates and sulfarsenates of $\mathrm{Fe}$ is shown to be a significant

Table 1

Representative chemical analyses of cassiterite and its exsolutions from the abandoned Ervedosa mine

\begin{tabular}{|c|c|c|c|c|c|}
\hline & \multicolumn{2}{|c|}{ Cassiterite } & \multirow[t]{2}{*}{ Titanian-Ixiolite } & \multirow[t]{2}{*}{ Wolframo-Ixiolite } & \multirow[t]{2}{*}{ Ferro-Columbite } \\
\hline & lighter & darker & & & \\
\hline $\mathrm{SnO}_{2}$ & 99.70 & 98.87 & 0.69 & 0.39 & 1.06 \\
\hline $\mathrm{Nb}_{2} \mathrm{O}_{5}$ & 0.01 & 0.26 & 60.92 & 57.19 & 13.45 \\
\hline $\mathrm{Ta}_{2} \mathrm{O}_{5}$ & 0.05 & 0.20 & 9.89 & 11.74 & 63.05 \\
\hline $\mathrm{TiO}_{2}$ & 0.11 & 0.18 & 5.10 & 3.29 & 2.60 \\
\hline $\mathrm{FeO}$ & 0.04 & 0.03 & 11.24 & 5.54 & 13.70 \\
\hline $\mathrm{MnO}$ & 0.05 & 0.04 & 8.36 & 12.80 & 4.50 \\
\hline $\mathrm{WO}_{3}$ & - & - & 3.37 & 7.79 & 1.05 \\
\hline Total & 99.96 & 99.58 & 99.57 & 98.74 & 99.41 \\
\hline
\end{tabular}


Table 2

Representative chemical analyses of sulphides and sulphosalts from the abandoned Ervedosa mine

\begin{tabular}{|c|c|c|c|c|c|c|c|c|}
\hline & Pyrrotite & Pyrite & Arsenopyrite & Sphalerite & Chalcopyrite & Stannite & Bismuthinite & Matildite \\
\hline $\mathrm{Cu}$ & 0.14 & - & 0.13 & 0.04 & 33.38 & 28.47 & 0.42 & - \\
\hline $\mathrm{Ag}$ & - & 0.06 & 0.08 & 0.01 & 0.04 & 0.18 & 0.20 & 24.25 \\
\hline $\mathrm{Zn}$ & 0.05 & 0.09 & 0.07 & 60.23 & 0.05 & 1.33 & 0.12 & 0.13 \\
\hline $\mathrm{Fe}$ & 60.15 & 46.30 & 34.69 & 4.50 & 30.28 & 12.01 & 0.45 & 1.70 \\
\hline $\mathrm{Mn}$ & 0.02 & 0.05 & 0.02 & 0.05 & 0.01 & 0.03 & 0.10 & - \\
\hline $\mathrm{Cd}$ & - & - & - & 0.76 & - & 0.08 & - & - \\
\hline $\mathrm{Sb}$ & - & 0.04 & 0.07 & - & - & - & - & - \\
\hline As & 0.12 & 0.30 & 45.58 & 0.03 & 0.02 & 0.06 & - & - \\
\hline $\mathrm{Bi}$ & - & - & - & - & - & - & 79.08 & 54.64 \\
\hline $\mathrm{Pb}$ & - & - & - & - & - & - & 0.78 & 2.50 \\
\hline $\mathrm{Sn}$ & - & - & - & - & - & 29.80 & - & - \\
\hline $\mathrm{S}$ & 39.19 & 52.85 & 19.48 & 34.05 & 35.84 & 27.48 & 19.10 & 16.67 \\
\hline Total & 99.67 & 99.69 & 100.12 & 99.67 & 99.62 & 99.44 & 100.25 & 99.89 \\
\hline
\end{tabular}
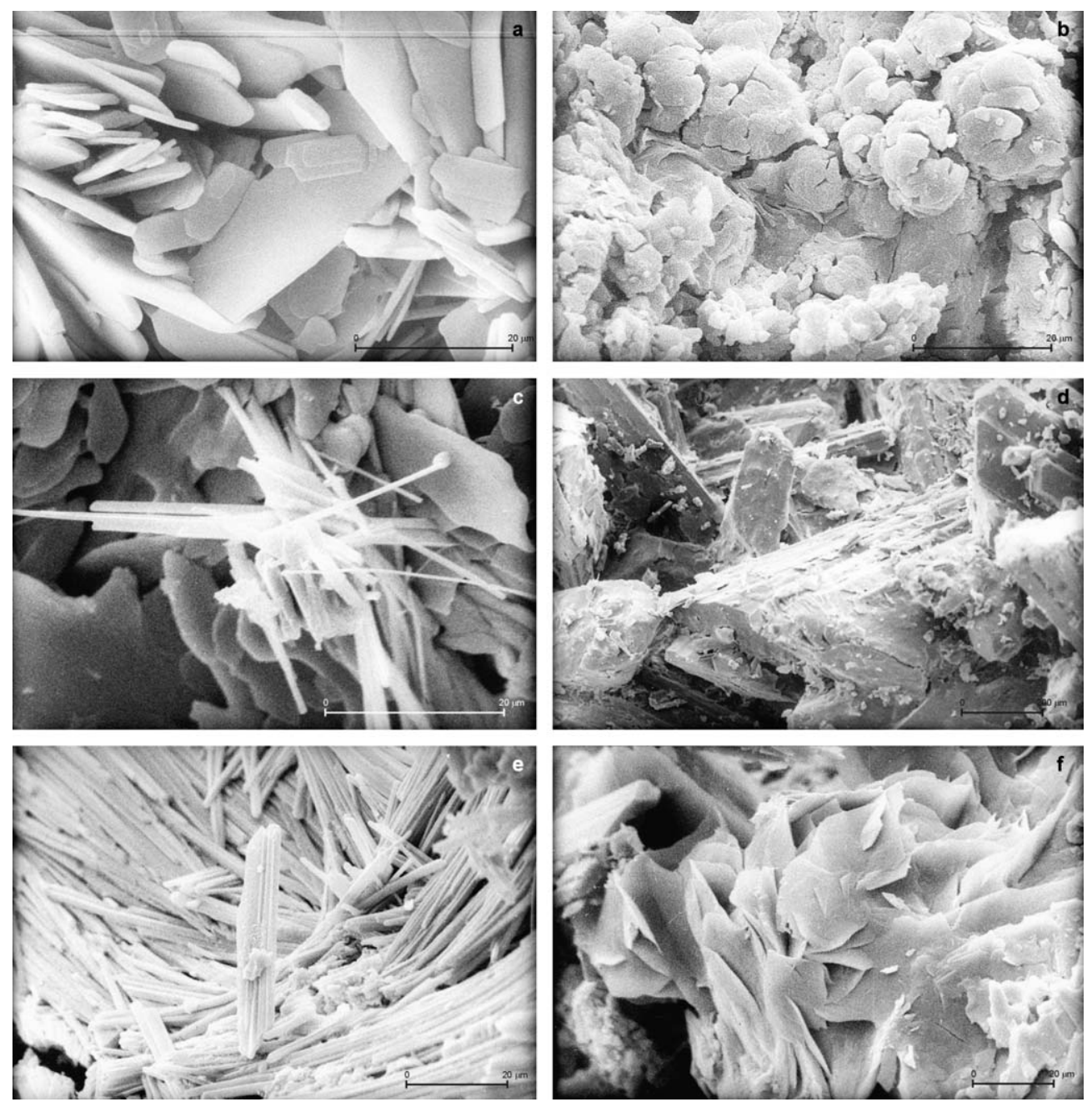

Fig. 3. Back-scattered electron images obtained on SEM. (a) copiapite; (b) aluminocopiapite; (c) halotrichite, (d) gypsum; (e) pickeringite and (f) alunogen. 
Table 3

Chemical compositions of AMD discharges and stream v ers inside and outside mine influence, from the Ervedosa abandoned mine

\begin{tabular}{|c|c|c|c|c|c|c|c|c|c|c|c|c|c|c|c|c|c|c|}
\hline \multirow[t]{2}{*}{ Sample } & \multirow[t]{2}{*}{ Type } & \multicolumn{3}{|c|}{ Field parameters } & \multirow{2}{*}{$\begin{array}{l}\text { Major } \\
\text { anion } \\
(\mathrm{mg} / \mathrm{L}) \\
\mathrm{SO}_{4}\end{array}$} & \multicolumn{4}{|l|}{$\begin{array}{l}\text { Major } \\
\text { cations } \\
(\mathrm{mg} / \mathrm{L})\end{array}$} & \multicolumn{9}{|l|}{$\begin{array}{l}\text { Trace } \\
\text { elements } \\
(\mu \mathrm{g} / \mathrm{L})\end{array}$} \\
\hline & & $\mathrm{pH}$ & $\begin{array}{l}\text { E.C. } \\
(\mu \mathrm{S} / \mathrm{cm})\end{array}$ & $T\left({ }^{\circ} \mathrm{C}\right)$ & & $\mathrm{Ca}$ & $\mathrm{Mg}$ & $\mathrm{Na}$ & K & $\mathrm{Fe}$ & $\mathrm{Mn}$ & $\mathrm{Zn}$ & $\mathrm{Cu}$ & $\mathrm{Pb}$ & As & $\mathrm{Cd}$ & Co & $\mathrm{Ni}$ \\
\hline 1 Jan & IMI & 7.03 & 66.1 & 7.0 & 4.10 & 4.23 & 0.92 & 5.48 & 1.03 & 81.0 & 5.00 & 298 & 37.5 & 5.00 & 4.46 & 3.58 & 0.61 & 7.00 \\
\hline 2 Jan & OMI & 5.51 & 30.1 & 10.9 & 5.20 & 0.69 & 1.49 & 1.83 & 0.46 & 216 & 2.50 & 108 & 10.0 & 6.00 & 1.80 & 1.30 & 0.76 & 9.00 \\
\hline 3 Jan & OMI & 6.82 & 28.2 & 9.0 & 3.00 & 1.08 & 1.12 & 2.21 & 0.39 & 185 & 5.80 & 147 & 22.0 & 6.00 & 1.73 & 1.76 & 0.74 & 7.00 \\
\hline 4 Jan & OMI & 6.29 & 34.3 & 8.0 & 4.00 & 1.62 & 1.05 & 2.99 & 0.65 & 50.0 & 3.00 & 126 & 13.0 & 4.00 & 0.73 & 1.51 & 0.49 & 7.00 \\
\hline 5 Jan & OMI & 7.00 & 42.7 & 10.0 & 3.50 & 2.07 & 1.21 & 3.77 & 0.79 & 85.0 & 3.20 & 119 & 20.0 & 4.00 & 1.23 & 1.43 & 0.48 & 6.00 \\
\hline 6 Jan & AMD & 3.15 & 469 & 11.7 & 97.0 & 14.5 & 4.84 & 5.04 & 0.91 & 11200 & 45.0 & 1919 & 415 & 4.00 & 7.33 & 76.8 & 49.0 & 84.0 \\
\hline 7 Jan & AMD & 3.14 & 710 & 13.5 & 64.0 & 55.1 & 19.8 & 6.62 & 1.60 & 7220 & 720 & 8370 & 411 & 3.00 & 328 & 419 & 118 & 95.0 \\
\hline 8 Jan & AMD & 4.07 & 958 & 11.6 & 163 & 108 & 63.5 & 12.9 & 4.94 & 79.0 & 484 & 4173 & 395 & 3.00 & 36.2 & 167 & 331 & 97.0 \\
\hline 9 Jan & IMI & 4.38 & 827 & 10.8 & 20.0 & 95.9 & 52.3 & 13.3 & 3.78 & 18.0 & 22.0 & 2562 & 393 & 4.00 & 25.5 & 64.1 & 106 & 143 \\
\hline 10 Jan & OMI & 5.45 & 57.6 & 10.6 & 4.00 & 3.12 & 2.20 & 3.20 & 0.46 & 18.0 & 5.00 & 324 & 141 & 3.00 & 2.39 & 0.39 & 2.00 & 52.0 \\
\hline $11 \mathrm{Jan}$ & IMI & 4.56 & 186.7 & 10.4 & 7.00 & 12.4 & 9.23 & 3.33 & 0.81 & 108 & 5.3 & 1365 & 382 & 3.00 & 32.5 & 19.11 & 44.0 & 136 \\
\hline 12 Jan & OMI/TR & 6.30 & 94.9 & 10.4 & 6.00 & 4.71 & 2.86 & 2.44 & 0.35 & 96.0 & 1.70 & 109 & 83.0 & 2.00 & 0.61 & 0.13 & 0.60 & 23.0 \\
\hline 13 Jan & IMI/TR & 6.57 & 61.8 & 8.2 & 9.00 & 5.45 & 3.17 & 2.32 & 0.32 & 84.0 & 13.0 & 230 & 63.0 & 3.00 & 0.50 & 3.22 & 3.00 & 18.0 \\
\hline 14 Jan & IMI/TR & 6.49 & 55.0 & 8.4 & 8.00 & 4.91 & 3.00 & 2.22 & 0.32 & 76.0 & 2.00 & 134 & 20.0 & 3.00 & 0.45 & 1.61 & 0.39 & 9.00 \\
\hline 15 Jan & IMI/TR & 7.02 & 54.6 & 8.7 & 7.00 & 4.92 & 2.95 & 2.30 & 0.32 & 88.0 & 27.0 & 155 & 15.0 & 3.00 & 0.64 & 0.19 & 0.64 & 9.00 \\
\hline $1 \mathrm{Mar}$ & IMI & 6.26 & 48.6 & 12.0 & 5.00 & 17.6 & 0.74 & 4.51 & 0.88 & 91.0 & 4.10 & 293 & 7.00 & 4.00 & 3.95 & 3.52 & 0.55 & 13.0 \\
\hline 3 Mar & OMI & 6.35 & 28.1 & 10.8 & 3.20 & 0.99 & 1.13 & 2.50 & 0.39 & 307 & 4.00 & 128 & 7.00 & 3.00 & 3.28 & 1.54 & 0.71 & 9.00 \\
\hline 4 Mar & OMI & 6.62 & 33.1 & 10.3 & 3.20 & 0.74 & 1.09 & 3.15 & 0.63 & 411 & 5.30 & 116 & 9.00 & 1.00 & 0.32 & 1.39 & 3.00 & 5.99 \\
\hline 5 Mar & OMI & 6.98 & 42.9 & 15.1 & 5.00 & 2.10 & 1.24 & 4.18 & 0.72 & 64.0 & 2.70 & 138 & 178 & 5.00 & 2.09 & 1.66 & 3.00 & 7.00 \\
\hline 6 Mar & AMD & 3.53 & 130 & 12.4 & 13.4 & 1.90 & 0.51 & 4.29 & 0.55 & 922 & 9.00 & 286 & 410 & 3.00 & 2.77 & 11.4 & 6.12 & 26.0 \\
\hline 7 Mar & AMD & 3.07 & 726 & 15.1 & 72.2 & 46.6 & 16.6 & 8.17 & 1.57 & 6750 & 908 & 6226 & 410 & 5.00 & 378 & 311 & 112 & 89.0 \\
\hline $8 \mathrm{Mar}$ & AMD & 4.00 & 984 & 12.8 & 111 & 138 & 68.5 & 14.4 & 4.70 & 101 & 260 & 3688 & 296 & 3.00 & 48.0 & 148 & 396 & 106 \\
\hline 9 Mar & IMI & 5.20 & 730 & 12.5 & 17.6 & 85.4 & 58.1 & 14.0 & 2.98 & 25.0 & 38.0 & 1193 & 100 & 10.0 & 16.8 & 29.8 & 78.9 & 174 \\
\hline $10 \mathrm{Mar}$ & OMI & 5.57 & 42.7 & 12.8 & 3.40 & 2.04 & 1.44 & 3.04 & 0.39 & 50.0 & 1.30 & 245 & 74.0 & 4.00 & 3.54 & 0.29 & 2.46 & 38.0 \\
\hline $11 \mathrm{Mar}$ & IMI & 4.43 & 194.6 & 12.6 & 9.81 & 13.6 & 9.22 & 3.72 & 0.95 & 40.0 & 2.3 & 1482 & 800 & 1.00 & 113 & 20.75 & 51.4 & 170 \\
\hline $12 \mathrm{Mar}$ & OMI/TR & 6.58 & 61.7 & 12.3 & 5.80 & 4.57 & 2.81 & 2.24 & 0.33 & 120 & 0.00 & 168 & 16.0 & 1.00 & 0.59 & 0.20 & 2.00 & 5.00 \\
\hline 13 Mar & IMI/TR & 6.45 & 65.7 & 12.9 & 9.40 & 4.85 & 2.94 & 2.15 & 0.31 & 83.0 & 64.0 & 142 & 10.0 & 3.00 & 0.25 & 1.99 & 3.00 & 11.0 \\
\hline 14 Mar & IMI/TR & 6.82 & 51.9 & 13.5 & 7.70 & 4.70 & 2.93 & 2.17 & 0.29 & 87.0 & 19.0 & 139 & 8.00 & 3.00 & 0.36 & 1.67 & 0.40 & 9.00 \\
\hline 15 Mar & IMI/TR & 7.33 & 52.1 & 14.0 & 6.50 & 4.65 & 2.92 & 2.24 & 0.31 & 366 & 51.0 & 132 & 6.00 & 4.00 & 0.21 & 0.16 & 0.43 & 7.00 \\
\hline 3 May & OMI & 7.01 & 28.8 & 15.6 & 2.70 & 1.00 & 0.49 & 2.45 & 0.23 & 102 & 4.70 & 130 & 6.53 & 1.98 & 2.30 & 1.56 & 0.73 & 8.65 \\
\hline 4 May & OMI & 6.07 & 34.7 & 14.8 & 3.50 & 1.41 & 0.47 & 3.19 & 0.58 & 54.3 & 3.70 & 134 & 17.9 & 1.25 & 1.50 & 1.61 & 0.71 & 12.2 \\
\hline 5 May & OMI & 7.01 & 47.6 & 18.4 & 3.00 & 2.31 & 1.19 & 4.13 & 0.57 & 88.0 & 3.00 & 149 & 89 & 0.52 & 0.75 & 1.79 & 0.51 & 2.49 \\
\hline 6 May & AMD & 3.36 & 245 & 13.7 & 54.0 & 3.60 & 0.99 & 5.00 & 0.81 & 1603 & 200 & 266 & 242 & 1.15 & 3.65 & 10.6 & 34.9 & 12.5 \\
\hline 7 May & AMD & 3.24 & 673 & 16.0 & 38.0 & 26.0 & 15.0 & 6.84 & 1.64 & 3777 & 2959 & 3420 & 1893 & 1.90 & 257 & 171 & 263 & 150 \\
\hline
\end{tabular}




\begin{tabular}{|c|c|c|c|c|c|c|c|c|c|c|c|c|c|c|c|c|c|c|}
\hline 8 May & AMD & 4.17 & 1007 & 15.8 & 150 & 87.2 & 56.7 & 18.6 & 4.30 & 49.6 & 670 & 3770 & 3429 & 0.48 & 32.0 & 151 & 201 & 192 \\
\hline 9 May & IMI & 5.14 & 745 & 14.6 & 18.0 & 65.9 & 48.2 & 16.0 & 2.93 & 8.74 & 95.4 & 722 & 168 & 10.6 & 18.5 & 18.1 & 118 & 129 \\
\hline 11 May & IMI & 4.68 & 192.1 & 16.2 & 5.00 & 11.6 & 10.55 & 4.83 & 1.08 & 21.9 & 4.25 & 1519 & 553 & 0.35 & 20.5 & 21.27 & 33.4 & 156 \\
\hline 12 May & OMI/TR & 6.86 & 50.0 & 18.3 & 5.30 & 4.78 & 3.71 & 3.45 & 0.33 & 170 & 3.31 & 118 & 37.1 & 0.60 & b.1. & 0.14 & 0.56 & 49.1 \\
\hline 13 May & IMI/TR & 6.94 & 54.7 & 19.1 & 6.60 & 5.54 & 3.75 & 3.39 & 0.31 & 122 & 293 & 225 & 38.7 & 13.7 & b.l. & 3.15 & 0.63 & 23.3 \\
\hline 14 May & IMI/TR & 6.98 & 67.0 & 20.0 & 6.54 & 4.43 & 3.01 & 2.63 & 0.22 & 111 & 38.0 & 147 & 16.2 & 0.71 & b.l. & 1.77 & 0.60 & 32.5 \\
\hline 15 May & IMI/TR & 7.25 & 66.6 & 22.1 & 6.00 & 4.67 & 2.23 & 2.26 & 0.18 & 69.0 & 24.6 & 142 & 13.3 & 1.65 & 0.30 & 0.17 & 0.64 & 1.78 \\
\hline $3 \mathrm{Jul}$ & OMI & 6.70 & 33.5 & 17.5 & 2.00 & 1.19 & 0.91 & 3.34 & 0.52 & 320 & 9.40 & 148 & 16.5 & 7.89 & 2.50 & 1.77 & 0.64 & 9.15 \\
\hline $4 \mathrm{Jul}$ & OMI & 6.32 & 32.9 & 16.2 & 1.53 & 1.34 & 1.00 & 3.37 & 0.85 & 276 & 5.60 & 69.8 & 7.30 & 2.77 & 0.80 & 0.84 & 0.55 & 5.31 \\
\hline $7 \mathrm{Jul}$ & AMD & 3.22 & 634 & 16.5 & 288 & 29.0 & 16.5 & 9.31 & 1.88 & 3466 & 3603 & 4314 & 1504 & 25.6 & 279 & 216 & 242 & 161 \\
\hline 9 Jul & IMI & 5.48 & 839 & 16.5 & 10.8 & 61.2 & 47.0 & 18.1 & 3.01 & 19.6 & 65.9 & 751 & 188 & 13.1 & 14.2 & 18.8 & 59.6 & 138 \\
\hline $12 \mathrm{Jul}$ & OMI/TR & 7.40 & 37.9 & 21.8 & 4.04 & 4.85 & 4.51 & 3.33 & 0.34 & 159 & 3.66 & 92.4 & 104 & 0.25 & b.l. & 0.11 & 0.37 & 3.79 \\
\hline $13 \mathrm{Jul}$ & IMI/TR & 6.30 & 57.8 & 22.3 & 7.00 & 3.81 & 2.92 & 2.13 & 0.22 & 109 & 342 & 261 & 83.5 & 18.3 & 0.80 & 3.66 & 0.68 & 14.6 \\
\hline $14 \mathrm{Jul}$ & IMI/TR & 7.58 & 54.5 & 23.0 & 5.40 & 9.36 & 6.24 & 4.25 & 0.51 & 183 & 40.7 & 152 & 28.3 & 2.08 & b.l. & 1.83 & 0.42 & 11.9 \\
\hline $15 \mathrm{Jul}$ & IMI/TR & 7.46 & 38.9 & 27.0 & 5.00 & 4.26 & 3.04 & 2.38 & 0.24 & 138 & 37.9 & 35.7 & 13.7 & 1.62 & b.l. & 0.04 & 0.62 & 1.83 \\
\hline $3 \mathrm{Sep}$ & OMI & 6.40 & 32.2 & 15.1 & 3.10 & 1.50 & 1.10 & 3.26 & 0.55 & 298 & 18.0 & 140 & 17.7 & 6.75 & 3.43 & 1.68 & 0.70 & 10.3 \\
\hline 4 Sep & OMI & 6.14 & 33.0 & 19.2 & 1.72 & 1.10 & 1.31 & 3.24 & 0.90 & 446 & 19.0 & 84.3 & 7.89 & 3.55 & 1.25 & 1.01 & 0.65 & 5.46 \\
\hline 7 Sep & AMD & 3.04 & 785 & 15.4 & 459 & 72.6 & 17.11 & 6.42 & 2.11 & 45040 & 5432 & 10390 & 2546 & 64.5 & 430 & 520 & 296 & 170 \\
\hline 9 Sep & IMI & 5.05 & 850 & 17.9 & 27.5 & 97.6 & 58.70 & 18.50 & 2.85 & 21.3 & 100 & 2970 & 410 & 25.7 & 17.0 & 74.3 & 135 & 156 \\
\hline $12 \mathrm{Sep}$ & OMI/TR & 6.46 & 63.6 & 14.0 & 4.20 & 5.25 & 4.28 & 3.40 & 0.42 & 163 & 4.00 & 138 & 146 & 1.56 & 0.60 & 0.17 & 0.86 & 5.30 \\
\hline $13 \mathrm{Sep}$ & IMI/TR & 6.10 & 108.1 & 14.3 & 18.4 & 6.03 & 4.87 & 3.30 & 0.34 & 248 & 450 & 445 & 760 & 36.7 & 1.5 & 6.23 & 2.67 & 46.7 \\
\hline 14 Sep & IMI/TR & 6.35 & 109.4 & 13.8 & 15.0 & 9.20 & 7.71 & 4.50 & 0.59 & 197 & 48.2 & 347 & 35.0 & 4.67 & 0.40 & 4.16 & 1.98 & 36.8 \\
\hline $15 \mathrm{Sep}$ & IMI/TR & 7.00 & 97.0 & 14.6 & 8.00 & 8.01 & 3.28 & 2.67 & 0.32 & 157 & 39.3 & 199 & 15.0 & 4.67 & 0.75 & 0.24 & 0.97 & 13.2 \\
\hline $3 \mathrm{Nov}$ & OMI & 3.39 & 174.5 & 9.1 & 4.2 & 0.94 & 0.70 & 2.20 & 0.39 & 345 & b.l. & 169 & 34.3 & 6.8 & 3.67 & 2.02 & 1.67 & 24.2 \\
\hline $4 \mathrm{Nov}$ & OMI & 5.80 & 33.3 & 10.3 & 4.50 & 1.25 & 0.54 & 2.24 & 0.57 & 134 & b.1. & 97.6 & 9.32 & 4.35 & 1.78 & 1.17 & 0.24 & 6.23 \\
\hline $6 \mathrm{Nov}$ & AMD & 3.15 & 514 & 7.8 & 69.2 & 20.05 & 0.38 & 3.80 & 0.80 & 8045 & 68.4 & 2010 & 407 & 3.89 & 6.90 & 80.4 & 64.3 & 96.7 \\
\hline 7 Nov & AMD & 3.38 & 517 & 13.4 & 27.5 & 23.2 & 16.60 & 4.73 & 1.70 & 2700 & 599 & 3050 & 348 & 2.56 & 115 & 153 & 78.5 & 59.6 \\
\hline $8 \mathrm{Nov}$ & AMD & 5.93 & 576 & 11.7 & 57.0 & 41.3 & 45.20 & 12.50 & 4.10 & 42.2 & 42.3 & 3020 & 235 & 0.34 & 11.0 & 121 & 196 & 36.4 \\
\hline $9 \mathrm{Nov}$ & IMI & 5.90 & 574 & 11.6 & 14.1 & 33.5 & 36.50 & 11.00 & 2.32 & 16.8 & 18.2 & 1801 & 157 & 2.34 & 11.0 & 45.0 & 32.5 & 95.4 \\
\hline $10 \mathrm{Nov}$ & OMI & 5.59 & 47.8 & 10.4 & 3.00 & 2.51 & 0.62 & 2.52 & 0.41 & 23.7 & b.1. & 425 & 86.7 & 2.25 & 2.70 & 0.51 & 2.07 & 27.4 \\
\hline $11 \mathrm{Nov}$ & IMI & 4.52 & 190.9 & 10.8 & 5.21 & 5.50 & 6.64 & 2.76 & 0.76 & 34.1 & 2.10 & 1334 & 311 & 0.56 & 41.0 & 18.68 & 39.2 & 132 \\
\hline $12 \mathrm{Nov}$ & OMI/TR & 6.26 & 95.3 & 8.0 & 4.60 & 3.36 & 1.70 & 2.31 & 0.32 & 175 & b.l. & 163 & 65.3 & 2.02 & 0.40 & 0.20 & 0.54 & 19.4 \\
\hline $13 \mathrm{Nov}$ & IMI/TR & 7.21 & 39.8 & 8.0 & 6.80 & 2.85 & 2.58 & 2.10 & 0.20 & 53.2 & 35.0 & 221 & 65.0 & 2.01 & b.l. & 3.10 & 0.14 & 11.1 \\
\hline $14 \mathrm{Nov}$ & IMI/TR & 6.30 & 49.5 & 7.8 & 5.50 & 4.10 & 2.00 & 2.00 & 0.35 & 67.4 & 4.00 & 127 & 10.4 & 2.45 & 0.30 & 1.52 & 0.20 & 8.72 \\
\hline $15 \mathrm{Nov}$ & IMI/TR & 7.15 & 48.3 & 8.0 & 6.75 & 3.48 & 2.48 & 2.10 & 0.30 & 57.3 & 9.00 & 136 & 6.45 & 3.23 & 0.30 & 0.16 & 0.67 & 2.10 \\
\hline
\end{tabular}


mechanism in controlling the concentration of heavy metals in pore solutions of high-sulfide mine wastes (Gieré et al., 2003).

The pathways of $\mathrm{FeS}_{2}$ oxidation may be controlled either chemically or organically by microorganisms. The kind of sulphate mineral formed is a function of solution composition and local conditions, e.g. humidity, acidity and redox potential. The evolution of mineral composition of the sulphate efflorescences, during storage in the presence of caulinite generate halotrichite, which appears in the earlier stage of weathering and is then replaced by alunogen (Parafiniuk and Stepisiewicz, 1999). Kaolinite absorbs water, and is an indispensable agent in each oxidation mechanism (Parafiniuk and Stepisiewicz, 2000).

Oxidation processes operate most intensively in Ervedosa specimens which contain kaolinite impurities. Copiapite commonly appears on the surface of granitic rocks due to kaolinite clays which form very fine, granular, pale yellow encrustations. SEM images of copiapite display characteristic euhedral, flaky or platy forms (Fig. 3a), intergrown into rosette or bunch aggregates of aluminocopiapite (Fig. 3b). Halotrichite and pickeringite are the most frequently found minerals in the studied paragenesis. They form assemblages composed of hair-like or acicular, white or colourless transparent crystals up to a few $\mathrm{mm}$ long (Figs. 3c and e). Usually, the longer crystals grow perpendicular to the sulphide surface. Some crystals form tufted aggregates. Gypsum is also frequent in acicular crystals or in prismatic or fibrous fine-grained forms (Fig. 3d). Alunogen forms efflorescences of white, fibrous crys- tals on the sulphide specimens (Fig. 3f). Currently, alunogen is one of the main weathering products replacing halotrichite. Alunogen and metalunogen are commonly associated with muscovite granite with pyrite and in contact with black shades and Sn-bearing quartz veins.

\subsection{Geochemistry of waters}

With the aim of obtaining reference background data for stream waters outside the impact area of the mine, samples of natural stream water were collected at 6 points and analysed (Table 3 ). Three sampling points for AMD were located in drainage galleries and another 6 sampling points were located inside the impact area (Table 3). A classification based on $\mathrm{pH}$ and metal concentrations was proposed by Ficklin et al. (1992), considering that $\mathrm{Zn}, \mathrm{Cu}, \mathrm{Cd}$, $\mathrm{Ni}, \mathrm{Co}$ and $\mathrm{Pb}$ are the major heavy metals found in mine drainage samples (Fig. 4). The Ervedosa acid mine drainage is mainly acid/high metal, while the natural stream water outside the mine is mainly near-neutral/low metal. The higher $\mathrm{SO}_{4}$ and metal concentrations are usually found in the acid leachates from the galleries and waste piles (samples 6, 7 and 8), which indicate oxidation and subsequent dissolution of pyrite and the accompanying sulphides (chalcopyrite, sphalerite, arsenopyrite and pyrrothite). In general, these AMD compositions indicate concentrations of totally dissolved solids $\mathrm{SO}_{4}$ and metals) that are within the ranges reported for sulphides hosting mineralisation (Ficklin et al., 1992; Plumlee et al., 1992; Petrunic and Al, 2005; Sánchez España et al., 2005). The correlations between trace

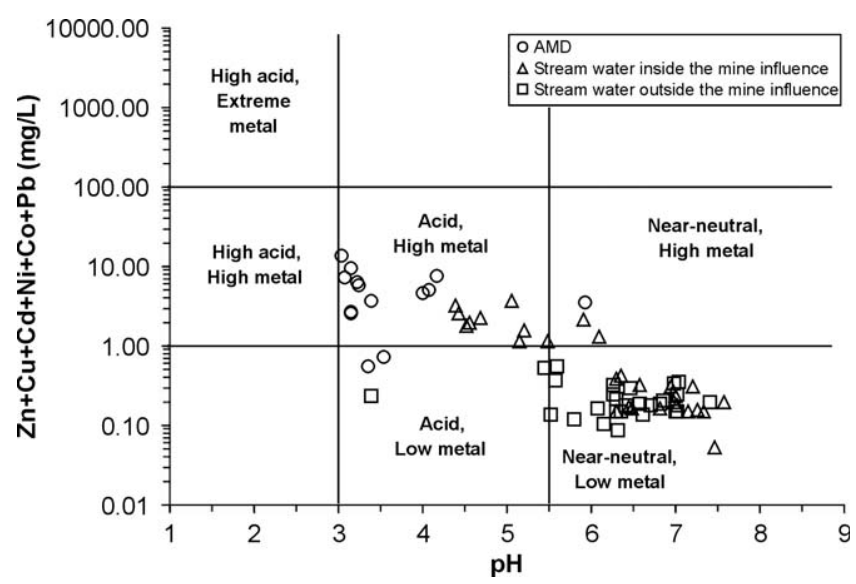

Fig. 4. Water samples from Ervedosa mine area plotted on the Ficklin et al. (1992) diagram based on pH and metal concentrations $(\mathrm{Zn}+\mathrm{Cu}+\mathrm{Cd}+\mathrm{Ni}+\mathrm{Co}+\mathrm{Pb})$. 
metals are poor and do not show any significant trend. The poor correlation between $\mathrm{As}$ and $\mathrm{Fe}$ and $\mathrm{Mn}$ was also found by Antunes et al. (2002). Nordstrom and Ball (1985) distinguish two types of behaviour of ions in acid mine drainage, depending on dissolution characteristics: non-conservative ions (e.g. As), which are rapidly eliminated during transport, and conservative ions (e.g. $\mathrm{SO}_{4}$ ) that are stable in solution and are the last to be adsorbed or precipitated. In the diagrams, all samples inside the mine's area of influence, including the samples collected in major flow rate areas (samples 12, 13,
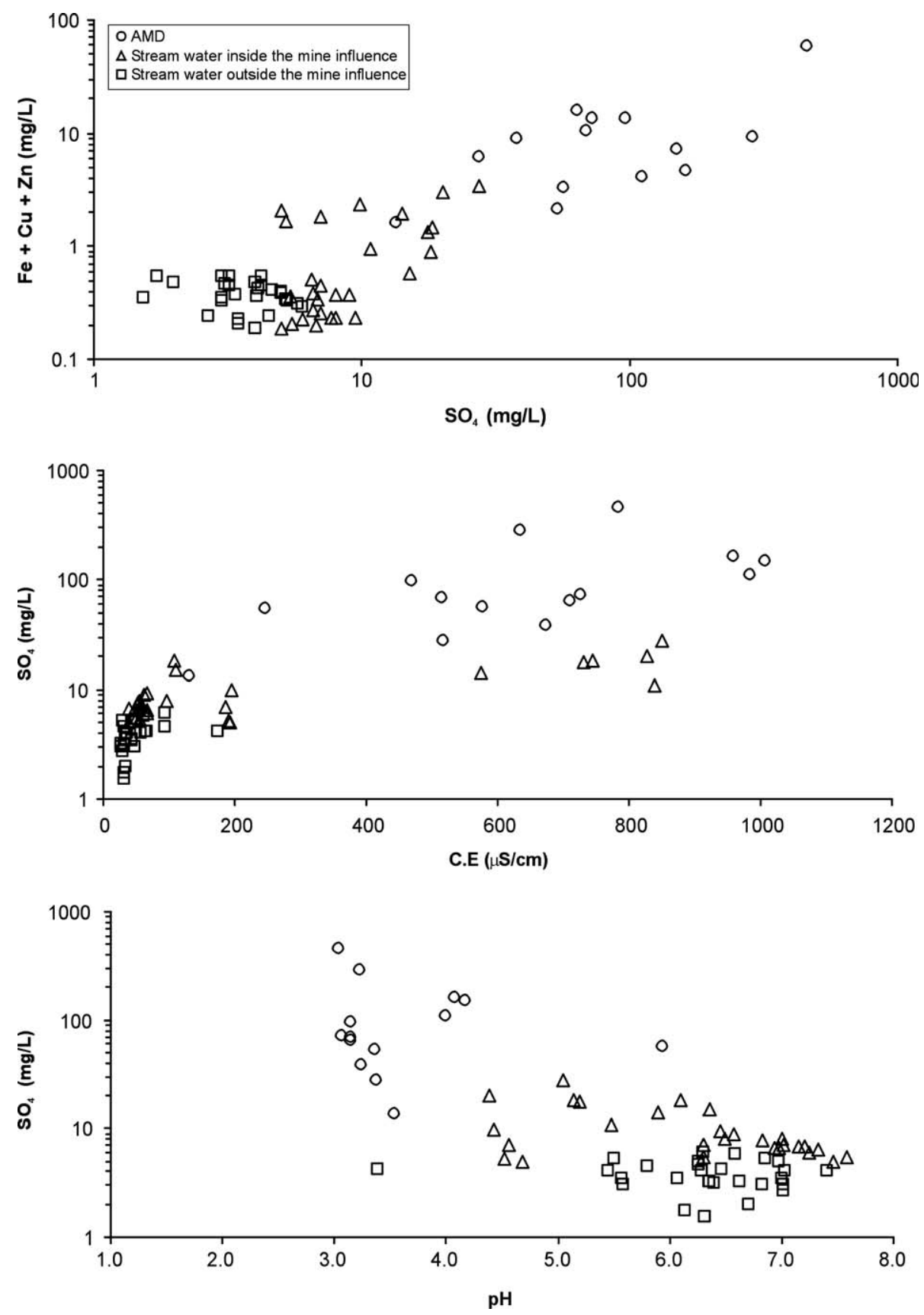

Fig. 5. (a) $(\mathrm{Fe}+\mathrm{Cu}+\mathrm{Zn})$ vs. $\mathrm{SO}_{4}$ diagram; (b) $\mathrm{SO}_{4}$ vs. C.E (electrical conductivity, EC) diagram; (c) $\mathrm{SO}_{4}$ vs. pH diagram, for $\mathrm{AMD}$, and stream waters inside the mine influence and outside the mine influence. 

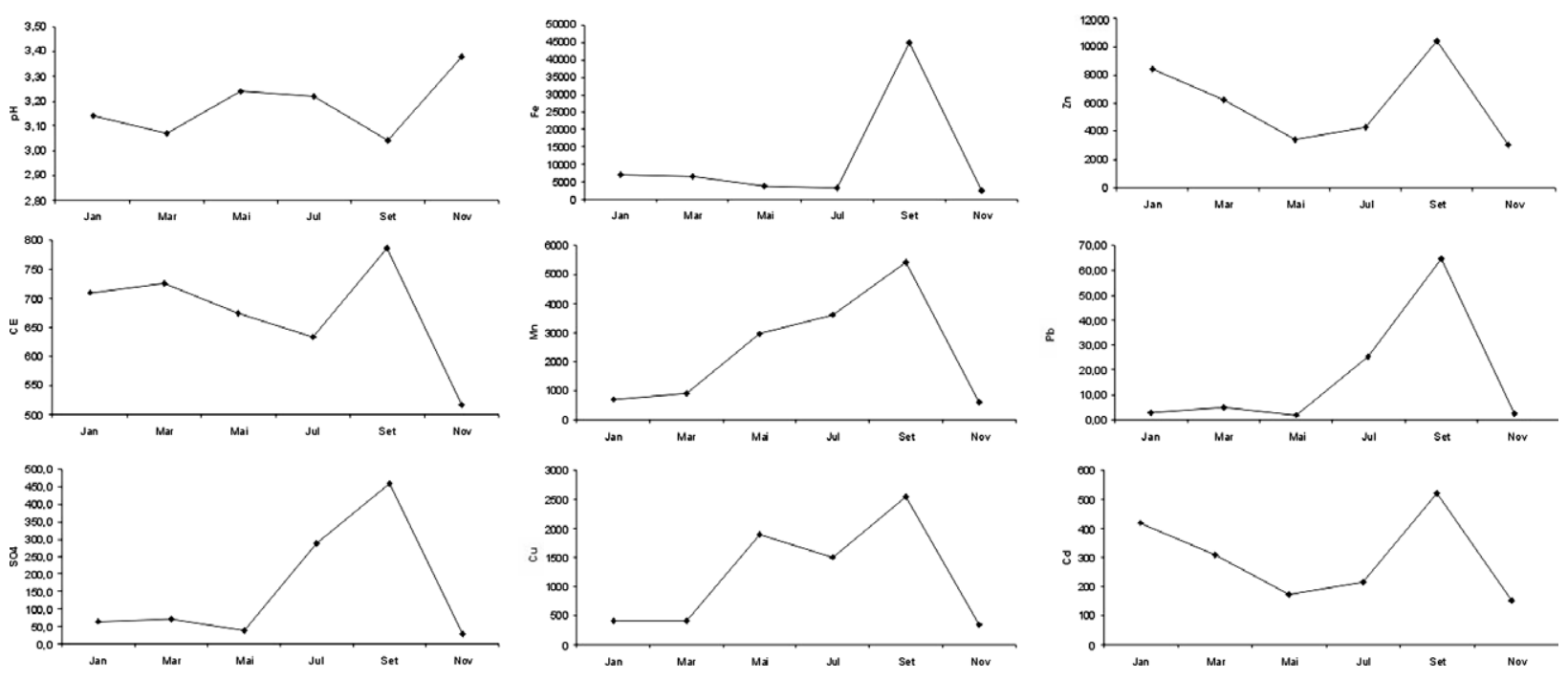

Fig. 6. Seasonal variations, over one year, in AMD (sample 7) composition for $\mathrm{pH}, \mathrm{SO}_{4}(\mathrm{mg} / \mathrm{L}), \mathrm{EC}(\mu \mathrm{S} / \mathrm{cm})$ and metal concentration (Fe, $\mathrm{Mn}, \mathrm{Cu}, \mathrm{Zn}, \mathrm{Pb}$ and $\mathrm{Cd}$, in $\mu \mathrm{g} / \mathrm{L})$.

14, 15 from Tuela River), show $\mathrm{SO}_{4}$ positive correlation for metals $(\mathrm{Fe}+\mathrm{Cu}+\mathrm{Zn})$ and Electrical Conductivity (EC) versus negative correlation for $\mathrm{pH}$ (Fig. 5). Samples from the Tuela River show $\mathrm{SO}_{4}$ contamination due to ion conservative behaviour. Toxic heavy metals show similar behavior to $\mathrm{SO}_{4}$, so the concentrations of these elements may be decreased by dilution.

AMD is controlled by the leaching process involving mineralised veins with sulphides. The hydrochemical features in the Ervedosa area appear to be dominated by the oxidation of Fe-bearing sulphide minerals (pyrite, pyrrhotite, arsenopyrite, chalcopyrite and sphalerite) producing $\mathrm{H}^{+}, \mathrm{SO}_{4}^{2-}$ and metals (Me) in solution (aq), as in the following reactions proposed by Cidu et al. (1997):

$$
\begin{aligned}
& (\mathrm{Fe}, \mathrm{Me}) \mathrm{S}_{2}+3.5 \mathrm{O}_{2}+\mathrm{H}_{2} \mathrm{O} \\
& \quad \rightarrow \mathrm{Fe}_{\mathrm{aq}}^{2+}, \mathrm{Me}_{\mathrm{aq}}^{2+}+2 \mathrm{SO}_{4}^{2-}+2 \mathrm{H}^{+} \\
& 2 \mathrm{Fe}_{\mathrm{aq}}^{2+}+0.5 \mathrm{O}_{2}+2 \mathrm{H}^{+} \rightarrow 2 \mathrm{Fe}_{\mathrm{aq}}^{3+}+\mathrm{H}_{2} \mathrm{O}
\end{aligned}
$$

There is a clear chemical distinction between granitic rock water drainage outside the mine impact area, the water inside this area, and the AMD-generated in the mine site. Superficial water outside the impact area has a $\mathrm{pH}$ close to neutral, low electrical conductivity and low ionic charge, while the drainage inside the mine's influence has progressively different characteristics. The water at the mining site is toxic and affected by AMD (pH 3.1-5.9), with high conductivity (130-1007 $\mu \mathrm{S} / \mathrm{cm})$ and significant As (3-
$430 \mu \mathrm{g} / \mathrm{L})$ and metal concentrations $(\mathrm{Cu}=235$ $3429 \mu \mathrm{g} / \mathrm{L}, \mathrm{Zn}=266-10390 \mu \mathrm{g} / \mathrm{L}, \mathrm{Fe}=42.2-45040$ $\mu \mathrm{g} / \mathrm{L}, \mathrm{Ni}=12.5-192 \mu \mathrm{g} / \mathrm{L}$ and $\mathrm{Co}=6.1-396 \mu \mathrm{g} / \mathrm{L}$ ), while in the surface granitic water outside the mine's influence (except anomalous sample 03-Nov) the $\mathrm{pH}$ measured is close to neutral ( $\mathrm{pH} 5.5-7$ ), with low conductivity $(28-95 \mu \mathrm{S} / \mathrm{cm})$ and As $(0.3-3.5 \mu \mathrm{g} / \mathrm{L})$ and metal concentrations are lower $(\mathrm{Cu}=6.5-178 \mu \mathrm{g} / \mathrm{L}$, $\mathrm{Zn}=69.8-425 \mu \mathrm{g} / \mathrm{L}, \mathrm{Fe}=18-446 \mu \mathrm{g} / \mathrm{L}, \quad \mathrm{Ni}=2.5$ $52 \mu \mathrm{g} / \mathrm{L}$ and $\mathrm{Co}=0-3 \mu \mathrm{g} / \mathrm{L})$.

The seasonal variations in AMD compositions are generally registered because, in dry periods, $\mathrm{pH}$ is lower and $\mathrm{EC}, \mathrm{SO}_{4}$ and, in consequence, metal concentration ( $\mathrm{Fe}, \mathrm{Mn}, \mathrm{Cu}, \mathrm{Zn}, \mathrm{Pb}$ and $\mathrm{Cd}$ ) are higher (Table 3 and Fig. 6). Dilution decreases metal concentration and $\mathrm{EC}$, and increases $\mathrm{pH}$, during winter.

\section{Conclusions}

Cassiterite is the main Sn ore at the Ervedosa Sn mine and it shows oscillatory zoning. Its geochemistry shows that darker zones are richer in $\mathrm{Fe}, \mathrm{Nb}, \mathrm{Ta}$, $\mathrm{Ti}$ and poorer in Sn than the lighter zones. Exsolution blebs of ferrocolumbite, manganocolumbite, titanian ixiolite, rutile, ilmenite and rare wolframite were found in the darker zones. Arsenopyrite is the most abundant sulphide and contains inclusions of pyrrhotite, bismuth, bismuthinite and matildite. Other sulphides are pyrite, sphalerite, chalcopyrite and stannite. Some mineralised veins and schist and granite wallrocks are in close contact with 
Sn-bearing quartz veins, secondarily consisting of mixtures of $\mathrm{Ca}, \mathrm{Al}, \mathrm{Fe}$ and $\mathrm{Mg}$ hydrated sulphates such as pickeringite, copiapite, halotrichite, alunogen, gypsum. On the other hand, oxides, hydroxides, arsenates and residual major mineral phases (albite, muscovite and quartz) occur in mine tailings.

The Ervedosa acid mine drainage is mainly acid/ high metal, while the natural stream waters outside its impact area are mainly near-neutral/low metal and the drainage inside this area has intermediate characteristics. Sulphate is a conservative ion and shows positive correlation with metals $(\mathrm{Fe}+\mathrm{Cu}+$ $\mathrm{Zn})$ and $\mathrm{EC}$, and a negative correlation with $\mathrm{pH}$. All AMD samples inside the mine impact area, including the samples collected in major flow rate zones, show $\mathrm{SO}_{4}$ contamination. Surface water outside the mine influence has $\mathrm{pH}$ close to neutral, low EC $(28-95 \mu \mathrm{S} / \mathrm{cm})$ and low ionic charge. The waters drained by galleries and tailings are toxic and affected by AMD ( $\mathrm{pH}=3.1-5.9)$, with high conductivity $(130-1007 \mu \mathrm{S} / \mathrm{cm})$ and significant As (3-430 $\mu \mathrm{g} / \mathrm{L})$ and metal concentrations $(\mathrm{Cu}=$ $235-3429 \mu \mathrm{g} / \mathrm{L}, \mathrm{Zn}=266-10390 \mu \mathrm{g} / \mathrm{L}, \mathrm{Fe}=42.2$ $45040 \mu \mathrm{g} / \mathrm{L}, \quad \mathrm{Ni}=12.5-192 \mu \mathrm{g} / \mathrm{L}$ and $\mathrm{Co}=6.1-$ $396 \mu \mathrm{g} / \mathrm{L}$ ), while the water inside the mine has intermediate values.

Waters associated directly with mineralised drainage veins must not be used for human consumption and agriculture. The concentrations of $\mathrm{Fe}, \mathrm{Zn}, \mathrm{Cu}, \mathrm{Cd}$ are generally greater than those allowed by current drinking-water regulations and sometimes above values recommended for agriculture (EU Council Directive 75/440/EEC of 16 June 1975 and Portuguese law, 1998, Decree No. 236/98, concerning the quality required of surface water intended for the abstraction of drinking water and agricultural activities).

\section{Acknowledgements}

Thanks are due to Prof. M. R. Machado Leite, Dr. J.M.F. Ramos for providing access to electron microprobe facilities at the INETI (ex. Instituto Geológico e Mineiro) S. Mamede de Infesta, Portugal; Prof. Pedro Tavares and Eng. Nuno Martins at UTAD and Eng. Fernanda Guimarães at the University of Minho for providing access to scanning electron microscope facilities and to Prof. J. S. Pratas and Eng. Carlos Paulo from the University of Coimbra for the water analyses. This research was carried out under the programme of Geosciences Centre, Coimbra University, Portugal.

\section{References}

Antunes, I.M.H.R., Neiva, A.M.R., Silva, M.M.V.G., 2002. The mineralized veins and the impact of old mine workings on the environment at Segura, central Portugal. Chem. Geol. 190, 417-431.

APHA-AWWA-WPCF, 1995. Standard Methods for Examination of Water and Wastewater, 19. American Public Health Organisation, Washington.

Ávila, P.H.F., 2002. Dispersão de elementos vestigiais na envolvente da mina abandonada do Vale das Gatas (Sabrosa, Norte de Portugal): Implicações Ambientais. Unpubl. PhD Univ. Aveiro.

Bednar, A.J., Garbarino, J.R., Ranville, J.F., Wildeman, T.R., 2004. Effects of iron on arsenic speciation and redox chemistry in acid mine water. J. Geochem. Explor. 85, 55-62.

Beetsma, J.J., 1995. The late proterozoic/paleozoic and hercynian crustal evolution of the Iberian massif, N Portugal: as traced by geochemistry and $\mathrm{Sr}-\mathrm{Nd}-\mathrm{Pb}$ isotope systematics of pre-Hercynian terrigenous sediments and Hercynian granitoids. Ph Thesis, Vrije Univ.

Bigham, J.M., Schwertmann, U., Carlson, L., Murad, E., 1996. Schwertmannite and the chemical modeling of iron in acid sulphate waters. Geochim. Cosmochim. Acta 60, 2111-2121.

Cidu, R., Caboi, R., Fanfani, L., Frau, F., 1997. Acid drainage from sulfides hosting gold mineralization (Furtei, Sardinia). Environ. Geol. 30, 231-237.

Ficklin, W.H., Plumlee, G.S., Smith, K.S., McHugh, J.B., 1992. Geochemical classification of mine drainages and natural drainages in mineralized areas:. In: Kharaka, Y.K., Maest, A.S. (Eds.), Water-Rock Interaction, vol.7. Balkema, Rotterdam, pp. 81-384.

Gieré, R., Sidenko, N.V., Lazareva, E.V., 2003. The role of secondary minerals in controlling the migration of arsenic and metals from high-sulfide wastes (Berikul gold mine, Siberia). Appl. Geochem. 18, 1347-1359.

Gomes, M.E.P., Neiva, A.M., 2001. Mineralogical and geochemical characteristics of tin-bearing quartz veins, Ervedosa deposit, Northern Portugal. Boletin de la Socied. Esp. de Mineralogia 24, 99-116.

Gomes, M.E.P., Neiva, A.M., 2002. Petrogenesis of tin-bearing granites from Ervedosa, northern Portugal: the importance of magmatic processes. Chemie de Erde 62, 47-72.

Lee, P.K., Kang, M.J., Choi, S.H., Touray, J.C., 2005. Sulfide oxidation and the natural attenuation of arsenic and tarce metals in the waste rocks of the abandoned Seobo tungsten mine, Korea. Appl. Geochem. 20, 1687-1703.

Nordstrom, D.K., Ball, J.W., 1985. Toxic element composition of acid mine waters from sulfide ore depositsII Mine Water Congress, vol. 2. IMWA, Granada, pp. 749-757.

Oliveira, J.M.S., Farinha, J., Matos, J.X., Ávila, P., Rosa, C., Machado, M.J.C., Daniel, F.S., Martins, L., Leite, M.R.M., 2002. Diagnóstico ambiental das principais áreas mineiras degradadas do país. Boletim de Minas 39, 67-85.

Parafiniuk, J., Stepisiewicz, M., 1999. Sulphate minerals forming on pyrite and marcasite specimens stored under room conditions. Mineralogia Polonica 30, 59-72. 
Parafiniuk, J., Stepisiewicz, M., 2000. Available from: <http:// www.geo.uw.edu.pl/ZASOBY/PYRITE/pyrite.htm>.

Petrunic, B.M., Al, T.A., 2005. Mineral/water interaction in tailings from a tungsten mine, Mount Pleasant, New Brunswick. Geochim. Cosmochim. Acta 69, 2469-2483.

Plumlee, G.S., Smith, K.S., Ficklin, W.H., Briggs, P.H., 1992. Geological and geochemical controls on the composition of mine drainages and natural drainages in mineralized areas. In: Kharaka, Y.K., Maest, A.S. (Eds.), Water-Rock Interaction, vol.7. Balkema, Rotterdam, pp. 19-422.

Sánchez España, J., López Pamo, E., Santofimia, E., Aduvire, O., Reyes, J., Barettino, D., 2005. Acid mine drainage in the
Iberian Pyrite Belt (Odiel river watershed, Huelva, SW Spain). Appl. Geochem. 20, 1320-1356.

Valente, T.M.F., 2004. Modelos de caraterização de impacte ambiental para escombreiras reactivas- equílibrio e evolução de resíduos de actividade extractiva. Unpubl. $\mathrm{PhD}$, Univ. Minho.

Vetter, T.W., Pratt, K.W., Turk, G.C., Beck, C.M., Butler, T.A., 1995. Using instrumental techniques to increase the accuracy of the gravimetric determination of sulphate. Analyst 120, 2025-2032.

Webster, J.G., Swedlund, P.J., Webster, K.S., 1998. Trace metal adsorption onto acid mine drainage $\mathrm{Fe}(\mathrm{III})$ oxyhydroxysulphate. Environ. Sci. Technol. 32, 1361-1368. 